\title{
Una década de observatorios urbanos: los nuevos retos
}

\author{
Ricardo Villasís Keever ${ }^{1}$ - Zuridahi Rubio Rojo² - Ana María Delgadillo Silva³
}

\author{
Observatorio Urbano Local de San Luis Potosí
}

\begin{abstract}
RESUMEN
Los observatorios urbanos en México han pasado por una experiencia de más de una década. Actualmente los cambios a nivel mundial y nacional establecen condiciones para un reposicionamiento de las plataformas de indicadores urbanos. En el ámbito internacional ante la implementación de la Organización de las Naciones Unidas del programa de los Objetivos de Desarrollo Sostenible (ODS), el Objetivo 11 se dirige a las ciudades para mejorar las condiciones del hábitat humano; y en el ámbito nacional -en el marco de la nueva Ley General de Asentamientos Humanos- quedó instituida la figura de los Observatorios Urbanos, en estas condiciones existe una nueva oportunidad de los Observatorios para revalorar sus potencial y para incidir en políticas públicas, evaluando los procesos urbanos
\end{abstract}

\begin{abstract}
Urban observatories in Mexico have gone through an experience of more than a decade. Currently, the changes at the global and national levels establish conditions for a repositioning of urban indicators platforms. In the international context facing the implementation of the United Nations Organization of the Sustainable Development Goals (ODS) program, the Objective 11 is focused on the cities, to improve the conditions of the human habitat; And at the national scale -within the framework of the new General Law of Human Settlements-, the figure of the Urban Observatories was recognized. In these conditions, there is a new opportunity for Observatories to reveal their potential and to influence public policies, evaluating urban processes.
\end{abstract}

Palabras Clave: Observatorios urbanos, desarrollo sostenible, indicadores urbanos.

Keywords: Urban observatories, sustainable development, urban indicators.

El proceso de urbanización y el Observatorio Urbano Mundial

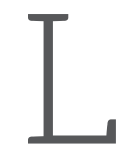

os antecedentes sobre indicadores urbanos se remontan a la Conferencia de Vancouver (1976) donde se expide La
Declaración de Asentamientos Humanos de Vancouver, conocida como HABITAT I, que creó la Agencia UN-HÁBITAT. En el documento de la conferencia se encuentran líneas de trabajo donde se detecta la necesidad de mayor intervención en las ciudades ante el reconoci-

1 Doctor en Ciencias Ambientales y Arquitecto. Responsable del Observatorio Urbano Local de San Luis Potosí. Facultad del Hábitat de la UASLP.oul@fh.uaslp.mx

2 Tesista de Maestría en Ciencias del Hábitat (MCH) de la UASLP.

3 Maestra en Ciencias del Hábitat y Arquitecta. Profesor-Investigador de la Facultad del Hábitat de la UASLP. 
miento de la inequidad del crecimiento económico que condena a millones de personas a la pobreza y a la insatisfacción de sus necesidades básicas, asimismo se manifiesta la importancia de los aspectos sociales, económicos, ecológicos y el deterioro del medio ambiente.

Fue hasta 1998 cuando la Agencia Hábitat cristaliza un esfuerzo sobre la metodología de indicadores urbanos, que fueron las bases de datos del Observatorio Urbano Global. Este ejercicio partió de la Conferencia de Estambul, Turquía, en 1996 a la que se le denominó Hábitat II, y que produjo La Declaración de Estambul sobre los Asentamientos Humanos, esta declaración consta de quince compromisos, que se resumen en dos ejes: a) vivienda adecuada para todos, y b) el desarrollo de los asentamientos humanos sostenibles en un mundo en proceso de urbanización.

Esta iniciativa, a nivel mundial, estableció un sistema de indicadores urbanos (Global Urban Observatory: GUO). Este sistema organiza el proceso de implementación de las bases de datos urbanos en dos clasificaciones: a) indicadores clave, agrupados en 20 unidades temáticas y que se expresan en número, porcentajes y en proporción; y b) datos cualitativos o checklists que informan sobre evaluación y que no pueden ser cuantificados. En ese momento, 53 ciudades latinoamericanas se sumaron al esfuerzo del GUO, sin pasar por los gobiernos centrales. De este modelo de indicadores se rescatan elementos importantes que pueden definir las características de los indicadores como instrumento metodológico: mensurabilidad, confiabilidad, comunicación, consistencia, comparabilidad.

La reproducción de observatorios locales en México durante los últimos años, hace evidente un mayor interés de diversas esferas en este tópico; tanto los gobiernos en sus tres órdenes, como las universidades, los centros de investigación y las redes institucionales, se han pronunciado a favor de replicar experiencias exitosas en esta materia. De igual forma, se han llevado a cabo continuamente congresos y eventos sobre el tema y se han generado diversas publicaciones y páginas web donde se pueden consultar los avances logrados en esta materia.

La proliferación de observatorios de diferentes tipos o categorías ha sido una actividad común en la última década (Villasís \& Moreno, 2008), así tenemos observatorios de seguridad, de movilidad, de medio ambiente, de la mujer, del mercado laboral, de violencia y género, entre otros y se han constituido en una herramienta útil para caracterizar y monitorear diferentes fenómenos, sin embargo su pertinencia depende del marco que sustenta su diseño ya que debe ciara respuesta al propósito por el cual se construye (Gudiño \& D'Inca, 2007:18).

Considero conveniente reproducir algunos de los escenarios que reporta la Agencia Hábitat de Naciones Unidas sobre la tendencia de urbanización en su más reciente publicación sobre el Informe Mundial de las Ciudades (UN-Hábitat, 2016).

- Mientras que en 1990 el 43 por ciento de la población mundial vivía en zonas urbanas, para el año 2015 la proporción había aumentado al 54 por ciento.

- El crecimiento más rápido son las ciudades medianas y pequeñas con menos de un millón habitantes, que representan el 59 por ciento de los población urbana del mundo

- La vivienda representa más del 70 por ciento del uso del suelo en la mayoría Determina la forma y las densidades urbanas

- Hoy el mundo es más desigual que hace veinte años, 75 por ciento de las ciudades del mundo tienen mayor desigualdad de ingreso que hace dos décadas.

De acuerdo con diversas investigaciones, algunas tendencias sobre la urbanización en el planeta indican que las ciudades más grandes del mundo estarán principalmente en el mundo en desarrollo y que las Mega ciudades, con más de 20 millones de habitantes, se están fortaleciendo en Asia, América Latina y África, además de que la escala del impacto ambiental de las ciudades será más significativo, y por otra parte, las ciudades en el mundo en desarrollo 
absorberán el 95 por ciento del crecimiento urbano total en las próximas dos décadas.

En resumen estamos ante un panorama de un mundo urbano, por lo que el propósito de establecer un modelo de referencia (o paquete de indicadores) para el desarrollo urbano que contribuye a los tomadores de decisiones para seleccionar alternativas, optimizar y gestionar programas y proyectos, identificar mejoras prácticas y experiencias con éxito en otros municipios; y además, servir a los ciudadanos para participar en el desarrollo, ejecución y control de las políticas correspondientes, entonces podremos caminar hacia un desarrollo más sostenible de las ciudades.

\section{Indicadores urbanos y monitoreo.}

Ahora bien, sobre el trabajo con indicadores urbanos y observatorios, podemos partir de una palabra clave: indicador. Este término proviene del latín indicare, que quiere decir mostrar o señalar una cosa, es decir que el indicador expresa algo más allá de sí mismo. Estas relaciones son complejas al involucrar distintas variables; para tal efecto se retoma la definición de Salvador Rueda (1999) sobre un sistema de indicadores urbanos, como un "... conjunto ordenado de variables sintéticas cuyo objetivo es proveer un visión totalizadora respecto a los intereses predominantes relativos a la realidad urbana de que se trate".

El uso de los indicadores tiene su empleo más difundido en el nivel económico (indicadores económicos), manejados por las agencias internacionales como el Banco Mundial o la OECD; o de otra naturaleza, como los indicadores de la Organización Mundial de la Salud. Con el interés de identificar mediante algunas variables las condiciones globales de los países en materias como el producto interno bruto (PIB), la población económicamente activa (PEA), el ingreso per cápita anual, natalidad y mortalidad, esperanza de vida, por mencionar algunos de ellos. Estas mediciones han establecido divisiones de los países con base en su riqueza, y también han determinado los niveles de financiamiento al desarrollo.
En todos estos casos el interés ha sido contar con una herramienta confiable para la toma de decisiones; sin embargo, se puede destacar que se tienen algunas dificultades, al pasar al nivel de desagregación de los datos de acuerdo a su escala: entidad federativa, región, municipio o localidad. Algunas desventajas que se han podido identificar en el proceso de construcción de indicadores urbanos, apuntan hacia la falta de consenso, también a la necesidad de grupos amplios de trabajo y recursos públicos disponibles; como limitaciones se encuentran la falta de información confiable y actualizada.

Como una oportunidad para los Observatorios, se puede apuntar que actualmente no existen evaluaciones de los planes de desarrollo urbano locales; que los indicadores y sistemas de indicadores son instrumentos más ágiles que los propios planes, y que permiten monitorear diversas variables espacial y temporalmente. Existen diversas metodologías sobre la evaluación de planes de desarrollo urbano (PDU) o planes de desarrollo territorial (POT), estas herramientas se basan fundamentalmente en el monitoreo. Las formas de evaluación tienen diferentes aproximaciones conceptuales que definen sus ejes de trabajo, así tenemos el caso de Camelo et al., (2014) que proponen la aplicación de diferentes niveles evaluativos que van desde los productos (proyectos) y la gestión hasta los impactos y los efectos.

Por otra parte se puede evaluar la sustentabilidad de los planes PDU o POT, como en el caso de la formulación de Flores (2014) que plantea el uso de criterios en los rubros específicos como participación y consulta ciudadana, la identidad, equidad y diversidad cultural, la ciudad compacta, funcional y mezcla de usos de suelo, y la integridad ecológica.

Otra perspectiva para evaluar los planes o los procesos urbanos es mediante un sistema de indicadores que sinteticen los alcances o desempeños, hasta la construcción de índices que impliquen la revisión total del sistema (Castro, 2002; Villasís, 2011). Otra faceta sobre los indicadores urbanos y los observatorios radica en que requieren de una etapa experimental, cuyos avances pudieran ser monitoreados por expertos en las diversas áreas de la observa- 
ción urbana.

Así, tenemos que las ventajas radican en la posibilidad de contar con un instrumento de monitoreo urbano ágil, con bases de datos confiables y con una simplificación del sistema, que lo hace útil para usuarios, aún para los no especializados; es una herramienta que puede participar en los procesos de toma de decisiones para los actores locales de la planeación. Es un modelo que puede aportar las diferencias entre ciudades, pero también al interior de la ciudad. En este caso se puede obtener la representación espacial a nivel $\mathrm{AGEB}^{4}$, ciudad, municipio o región, por lo anterior puede considerarse como un instrumento que permite el trabajo a diferentes escalas.

Estamos pues ante una tendencia generalizada hacia la construcción de observatorios, de toda índole, social, económica, competitividad, de violencia, de seguridad, es decir temáticas cada vez más diversificadas, por lo que es conveniente el fortalecimiento de los Observatorios Urbanos en México, que pueden considerarse como iniciadores de esta forma de trabajo del monitoreo con indicadores en nuestro país, con una década de experiencia y madurez; con un ámbito de intervención cada vez más amplio y aplicables en diversos rubros de investigación de interés para instituciones de educación superior, el Conacyt, las redes y los fondos estatales o regionales de investigación; en los tres niveles de gobierno y particularmente en los institutos municipales de planeación, con el abordaje de temas sobre la realidad urbana, la competitividad, la movilidad, la eficiencia urbana, la sostenibilidad, los catastros urbanos, la seguridad, la planeación urbana, estudios desde la perspectiva ciudadana, la perspectiva de género, los derechos humanos, pero también con indicadores en los poderes legislativo y judicial, entre otros.

Los nuevos retos: El cambio de los ODM por SDG

Para la Agenda Hábitat de las Naciones Uni- das, El Observatorio Urbano Mundial (Global Urban Observatory), desde sus inicios en 1994 logró una amplia cobertura del programa, mediante la Guía del Programa de Indicadores Urbanos ha recolectado información de más de 400 ciudades en el Mundo con cobertura en 130 países (Karl, 2001). El GUO -por sus siglas en inglés- es la plataforma más amplia a nivel mundial sobre indicadores urbanos, por lo anterior se puede considerar el modelo hegemónico, no obstante existen otras formas de mediciones urbanas en otras plataformas (Villasís, 2005). En México a partir del 2005 se implementaron los observatorios urbanos financiados por el Conacyt y la Sedesol, aunque ya existían otros ejercicios previos.

Los observatorios urbanos, han generado conocimiento relativo a la instrumentación de plataformas de investigación, análisis y resultados de indicadores urbanos, que expliquen el fenómeno urbano pero que también aporten una visión sistémica del monitorio urbano (Mendo, 2015). En términos generales el criterio que define a un indicador urbano está centrado en caracterizar algunas realidades urbanas específicas (o variables) de ciertas condiciones óptimas de bienestar de los ciudadanos y que permiten además la comparación global y eficaz de las áreas urbanas (Zoro, Morin, \& Bénié, 2003).

En el libro colectivo Observatorios Urbanos en México (Garrocho \& Álvarez, 2008), se aporta un capítulo sobre el análisis FODA sobre los Observatorios Urbanos en México (Mendo, 2008: 37-45), que sigue siendo vigente, se trata de las fortalezas, debilidades oportunidades y amenazas de los sistemas de monitoreo urbano; reconociendo que se han realizado diversas críticas sobre la construcción, la operación, su financiamiento y su pertinencia. El autor realizó un amplio repaso sobre las condiciones actuales de los sistemas de observación urbana en México, una de sus aseveraciones tiene que ver con criterios metodológicos y herramientas tecno científicas como uno de los elementos más consistentes en este proceso de observatorios urbanos, partiendo dos puntos positivos, el primero se refiere a que se ha adoptado

4 AGEB: Área geo-estadística básica (INEGI). 
un marco teórico común y el segundo radica en cuestiones materiales ya que se han equipado físicamente plataformas de observación, aunque la divulgación y las relaciones institucionales todavía son un reto todavía, aquí la cuestión financiera para su permanencia es un elemento no asegurado.

Con base en lo anterior se retoma el enfoque de la nueva oportunidad de los Observatorios Urbanos ante los grandes cambios que se han realizado en el marco de la operación y fundamentación de los observatorios y que se examinan a continuación en dos categorías o escalas, primero a nivel mundial y luego en México.

En el ámbito internacional, la Asamblea General de la Naciones Unidas, realizó un cambio de programa de los Objetivos de Desarrollo del Milenio (ODM), por los Objetivos de Desarrollo Sostenible (ODS, 2015), en el programa que se denominó Transformar nuestro mundo: la Agenda 2030 para el Desarrollo Sostenible, en el marco de una aspiración global de "...reconducir al mundo por el camino de la sostenibilidad y la resiliencia" (UN, 2015). Este programa incluyó 17 objetivos generales, dentro de los cuales, específicamente en materia de ciudades, se definieron los lineamientos para este tema mediante el reconocimiento que la gestión y el desarrollo sostenibles del medio urbano son fundamentales para la calidad de vida de nuestros pueblos, que dice textualmente:

Objetivo 11. Lograr que las ciudades y los asentamientos humanos sean inclusivos, seguros, resilientes y sostenibles

Para el seguimiento de ese objetivo, los gobiernos serán los principales responsables de realizar, el seguimiento y examen de los progresos conseguidos en el cumplimiento de los Objetivos y las metas, para ello se llevarán a cabo en un "proceso sistemático de seguimiento y examen en los distintos niveles..." (UN, 2015), cabe agregar que en el documento de los ODS se expresa que se están elaborando indicadores para contribuir a esa labor.

Por su parte, en México a partir de que se decretó La Ley General de Asentamientos Humanos, Ordenamiento Territorial y Desarrollo Urbano (DOF: 28/11/2016), la figura de los Observatorios Urbanos (y ciudadanos) quedó definida, ahora con la fuerza de la Ley. Dentro de los criterios establecidos, se propone la creación de institutos de planeación, observatorios ciudadanos, consejos participativos y otras estructuras institucionales y ciudadanas, y específicamente en el Capítulo Quinto, donde se determinan las funciones de los Observatorios Ciudadanos, que -por su importancia- se reproduce textualmente:

Artículo 99. Los gobiernos federal, de las entidades federativas, de los municipios y de las Demarcaciones Territoriales, promoverán la creación y funcionamiento de observatorios urbanos, con la asociación o participación plural de la sociedad, de las instituciones de investigación académica, de los colegios de profesionistas, de los organismos empresariales, de las organizaciones de la sociedad civil y el gobierno, para el estudio, investigación, organización y difusión de información y conocimientos sobre los problemas socio espaciales y los nuevos modelos de políticas urbanas y regionales y de gestión pública.

Los observatorios tendrán a su cargo las tareas de analizar la evolución de los fenómenos socios espaciales, en la escala, ámbito, sector o fenómeno que corresponda según sus objetivos, las políticas públicas en la materia, la difusión sistemática y periódica, a través e indicadores y sistemas de información geográfica de sus resultados e impactos.

Asimismo en artículo 100 de la mencionada Ley (LGAHOTDU), se definen las acciones tendientes al soporte para el funcionamiento de los observatorios, por parte de las dependencias y entidades de la Administración Pública Federal, Estatal, Municipal y de las Demarcaciones Territoriales, por su relevancia se trascribe literalmente:

I. Proporcionarles la información asequible sobre el proceso de Desarrollo Urbano y el ordenamiento territorial, así como de los actos administrativos y autorizaciones que afecten al mismo; 
II. Promover, desarrollar y difundir investigaciones, estudios, diagnósticos y propuestas en la materia;

III. Mejorar la recolección, manejo, análisis y uso de la información en la formulación de políticas urbanas;

IV. Estimular procesos de consulta y deliberación para ayudar a identificar e integrar las necesidades de información

V. Ayudar a desarrollar capacidades para la recolección, manejo y aplicaciones de información urbana, centrada en indicadores y mejores prácticas;

VI. Proveer información y análisis a todos los interesados para lograr una participación más efectiva en la toma de decisiones sobre Desarrollo Urbano y ordenamiento territorial;

VII. Compartir información y conocimientos con todos los interesados en el Desarrollo Urbano y el ordenamiento del territorio, y

VIII. Garantizar la interoperabilidad y la consulta pública remota de los sistemas de información.

Las entidades federativas establecerán las regulaciones específicas a que se sujetará la creación y operación de observatorios urbanos y para el ordenamiento territorial con base en esta Ley.

En las escalas mencionadas -mundial y nacional- quedó definido el nuevo marco de actuación de los Observatorios, primero en el ámbito internacional para las nuevas formas de evaluación de los sistemas urbanos mediante el seguimiento de los Objetivos del Desarrollo Sostenible; y en México con la nueva Ley de Asentamientos Humanos es clara en cuanto a la función de los Observatorios Urbanos; por lo anterior es evidente que la oportunidad ahora es formidable y que las plataformas de los Observatorios Urbanos deben estar preparados para asumir estos nuevos desafíos, aportando el nuevo conocimiento y metodologías pertinentes a los indicadores urbanos, sumando los recursos humanos de alto nivel, las plataformas tecnológicas y la infraestructura creada que debe ser competitiva a efecto de enfrentar las nuevas respuestas en un mundo cada vez más urbanizado, en consecuencia el proyecto de los observatorios urbanos deberá consolidarse en el futuro inmediato.

Nuevos retos se avizoran en este camino, uno de ellos es la capitalización, los recursos que se requieren para tener un sistema actualizado es un factor fundamental para el mantenimiento y operación de los Observatorios Urbanos. Así tenemos como posibles fuentes de ingreso los estudios y proyectos que requiere el sector público. La vinculación con las diversas instancias de para el desarrollo, la capacitación y gestión de los PDU y POT, pero también las publicaciones y productos SIG digitalizados, por otra parte el desarrollo de la investigación urbana con fondos concursables de agencias oficiales. Los productos derivados de estos esfuerzos servirán como base para la toma decisiones, formalizando ante las diversas instituciones estas herramientas, una de ellas la figura jurídica ahora incluida en la Ley, así como la representación formal de los Observatorios ante instituciones del sector público y privado. Especialmente con los gobierno federal, estatal y municipal, para incidir en políticas públicas, evaluando los procesos urbanos. Con la Agencia Hábitat de Naciones Unidas, como impulsor de este proyecto para contar con instrumentes más eficaces para el monitoreo del cumplimiento de las metas del desarrollo sostenible, explorando nuevas posibilidades.

\section{Referencias}

Camelo G., A.N., Solarte-Pazos \& L, López, O., (2015). Evaluación y seguimiento de planes de ordenamiento territorial en los municipios de Colombia. En: Sociedad y Economía No. 28, 2015 • pp. 163-180.

Castro B., M. (2002). Indicadores de desarrollo sostenible urbano. Una aplicación para Andalucía. Tesis Doctoral. Facultad de Ciencias Económicas y Empresariales, Universidad de Málaga. España.

D.O.F. (2016) La Ley General de Asentamientos Humanos, Ordenamiento Territorial y Desarrollo Urbano. Publicación en el Diario Oficial de la Federación. DOF: 28/11/2016. México.

Flores, L., M.L. (2014). La evaluación de la sustentabilidad de los planes urbanos. Un reto de construcción me- 
todológica: Montreal como caso de estudio. En: Montreal Sostenible.On theW@terfront. Núm.31, septiembre 2014 ISSN: 1139-736.

Garrocho, C. \& Álvarez, J. A. (Coord.). (2008). Observatorios Urbanos en México: lecciones, propuestas y desafíos. El Colegio Mexiquense, A.C., Zinacantepec, Estado de México, A.C.

Gudiño, M. E., \& D’Inca, V. (2007). Observatorio del medio ambiente urbano. Herramienta para el monitoreo y conservación de la biodiversidad local. En: Tiempo y Espacio, 14-16(17-19), 33-50.

Karl, G. (2001). Monitoring Implementation of the Habitat Agenda: The Global Urban Observatory. UN Chronicle, 38(1), 33.

Mendo-Gutiérrez, A. (2015). Observación urbana sistémica. Hacia una evaluación de ciudades desde la complejidad. Guadalajara, Jalisco: ITESO y Universidad de Guadalajara.

Rueda, S. (1999). Modelos e indicadores para ciudades más sostenibles. En: Taller sobre indicadores de Huella y calidad ambiental urbana. Agencia Europea del Medio Ambiente. Fundación Fórum Ambiental. España.

UN. (2015). Objetivos del Desarrollo Sostenible. 17 Objetivos para transformar nuestro mundo. [Disponible en: http://www.un.org/sustainabledevelopment/es/cities/ Fecha de consulta 20/08/2017]

UN-HABITAT (2016). World Cities Report 2016. Urbanization and Development: Emerging Futures. Abridged edition. First published 2016 by United Nations Human Settlements Programme (UN-HABITAT).

Villasís, R. (2005). Sobre indicadores de sustentabilidad urbana y observatorios. En: Revista Ciudades No. 68, pp. 21-28.

Villasís, R. (2011). Indicadores de sustentabilidad urbana: el caso de la zona metropolitana de San Luis Potosí. Tesis Doctoral. Facultades de Ciencias Químicas, Ingeniería y Medicina, Programa Multidisciplinario de Posgrado en Ciencias Ambientales Universidad Autónoma de San Luis Potosí.

Villasís, R. \& Moreno, A. (2008). "Observatorios urbanos y desarrollo territorial en San Luis Potosí: de la teoría a la realidad". En: Garrocho, C. y Álvarez, J. A. (Coord.), Observatorios Urbanos en México: lecciones, propuestas y desafíos, El Colegio Mexiquense, A.C., Zinacantepec, Estado de México, A.C.; cp., 145-176

Zoro, E., Morin, D. \& Bénié, G. (2003). Elaboration d'un indicateur de développement urbain (IDU). En : Canadian Journal Of Urban Research, 12(2), 320-343. 